Supporting Information for

\title{
A Nonheme Diiron Oxygenase Mimic That Generates a Diferric-Peroxo Intermediate Capable of Catalytic Olefin Epoxidation and Alkane Hydroxylation Including Cyclohexane
}

Williamson N. Oloo, ${ }^{\mathrm{a}}$ Miklós Szávuly, ${ }^{\mathrm{b}}$ József Kaizer $^{\#, \mathrm{~b}}$ and Lawrence Que, Jr. ${ }^{\ddagger}, \mathrm{O}$

a'Department of Chemistry, University of Minnesota, 207 Pleasant St. SE, Minneapolis, MN 55455, United States

${ }^{\mathrm{b}}$ Research Group of Bioorganic and Biocoordination Chemistry, University of Pannonia, 8200 Veszprém, Hungary 


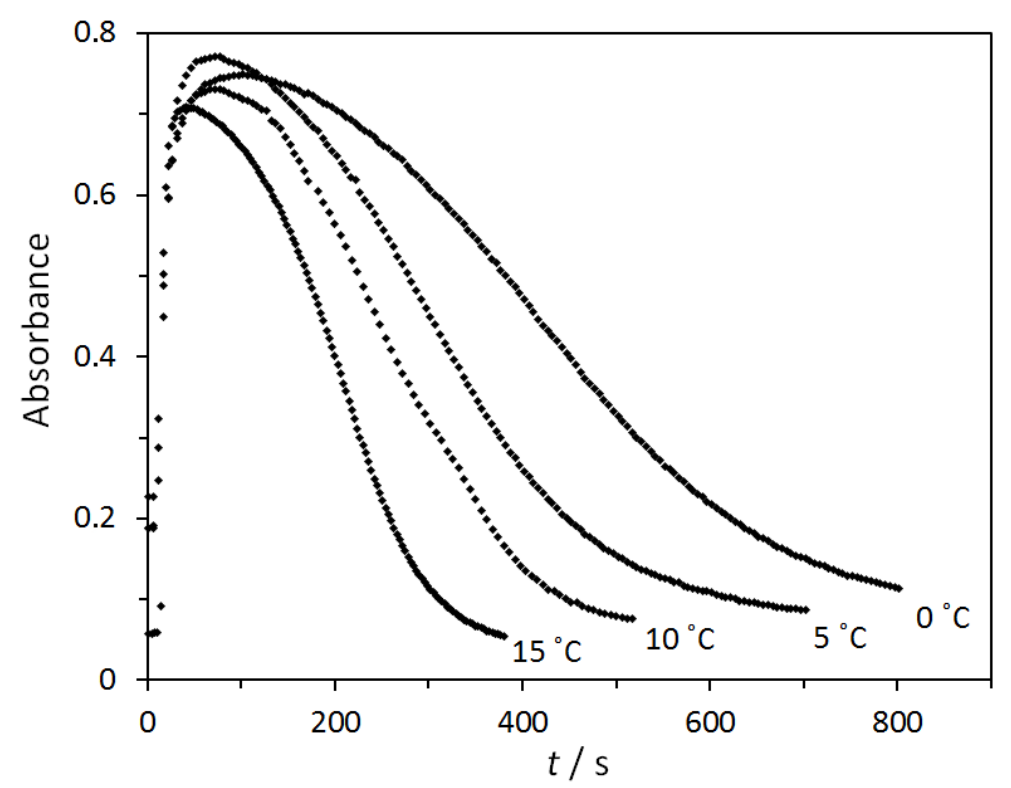

Figure S1. Temperature-dependent stability of 2. $[1]_{0}=10^{-3} \mathrm{M}$, in $\mathrm{MeCN}$ at $690 \mathrm{~nm}$.

\section{Materials}

All manipulations were performed under a pure argon atmosphere using standard Schlenktype inert-gas techniques unless otherwise stated. Solvents used for the reactions were purified by standard methods and stored under argon. Iron(II) perchlorate was purchased from commercial sources. The ligand 1,3-bis(2'-pyridylimino)isoindoline (indH) was prepared according to published procedures. ${ }^{10 a}$

\section{Analytical and physical measurements}

UV-vis spectra were recorded on an Agilent 8453 diode-array spectrophotometer using quartz cells. GC analyses were performed on an Agilent 7820A gas chromatograph equipped with a flame ionization detector and a $30 \mathrm{~m} \mathrm{HP-5.} \mathrm{GC-MS}$ analyses were carried out on a Shimadzu QP2010SE instrument equipped with a secondary electron multiplier detector with conversion dynode and a $30 \mathrm{~m}$ HP-5MS column.

\section{Stoichiometric oxidation of cyclohexanone with 2}

Reactions were carried out by mixing the substrate (cyclohexanone) with complex [1] $\left(10^{-3} \mathrm{M}\right)$ in $1.5 \mathrm{ml}$ acetonitrile and then adding 4 equiv. $\mathrm{H}_{2} \mathrm{O}_{2}$ at different temperatures $\left(0,5,1015^{\circ} \mathrm{C}\right)$. The reactions were then followed by UV-Vis spectroscopy at $680 \mathrm{~nm}$. 
Table S1. Kinetic data for the oxidation of cyclohexanone with 2

\begin{tabular}{|c|c|c|c|c|}
\hline $\begin{array}{l}{ }^{\circ} \mathrm{C} \\
{ }^{\circ} \mathrm{C}\end{array}$ & $\begin{array}{l}\text { [1] } \\
\left(10^{-3} \mathrm{M}\right)\end{array}$ & $\begin{array}{l}\text { [cyclohexanone }]_{0} \\
\text { (M) }\end{array}$ & $\begin{array}{l}k_{o b s} \\
\left(10^{-2} s^{-1}\right)\end{array}$ & $\begin{array}{l}k_{2}^{a} \\
\left(M^{-1} s^{-1}\right)\end{array}$ \\
\hline 5 & 1 & 0.05 & $1.92 \pm 0.06$ & $0.4 \pm 0.01$ \\
\hline 5 & 1 & 0.075 & $3.15 \pm 0.13$ & $0.38 \pm 0.02$ \\
\hline 5 & 1 & 0.15 & $5.91 \pm 0.18$ & $0.36 \pm 0.01$ \\
\hline 5 & 1 & 0.2 & $8.28 \pm 0.4$ & $0.4 \pm 0.02$ \\
\hline 5 & 1 & 0.075 & $3.15 \pm 0.06$ & $0.4 \pm 0.01$ \\
\hline 5 & 1.5 & 0.075 & $2.97 \pm 0.05$ & $0.34 \pm 0.02$ \\
\hline 5 & 2 & 0.075 & $2.72 \pm 0.04$ & $0.36 \pm 0.01$ \\
\hline 5 & 2.5 & 0.075 & $2.93 \pm 0.06$ & $0.37 \pm 0.02$ \\
\hline 0 & 1.0 & 0.075 & $2.6 \pm 0.07$ & $0.3 \pm 0.01$ \\
\hline 10 & 1 & 0.075 & $4.2 \pm 0.15$ & $0.51 \pm 0.03$ \\
\hline 15 & 1 & 0.075 & $5.1 \pm 0.16$ & $0.6 \pm 0.02$ \\
\hline
\end{tabular}




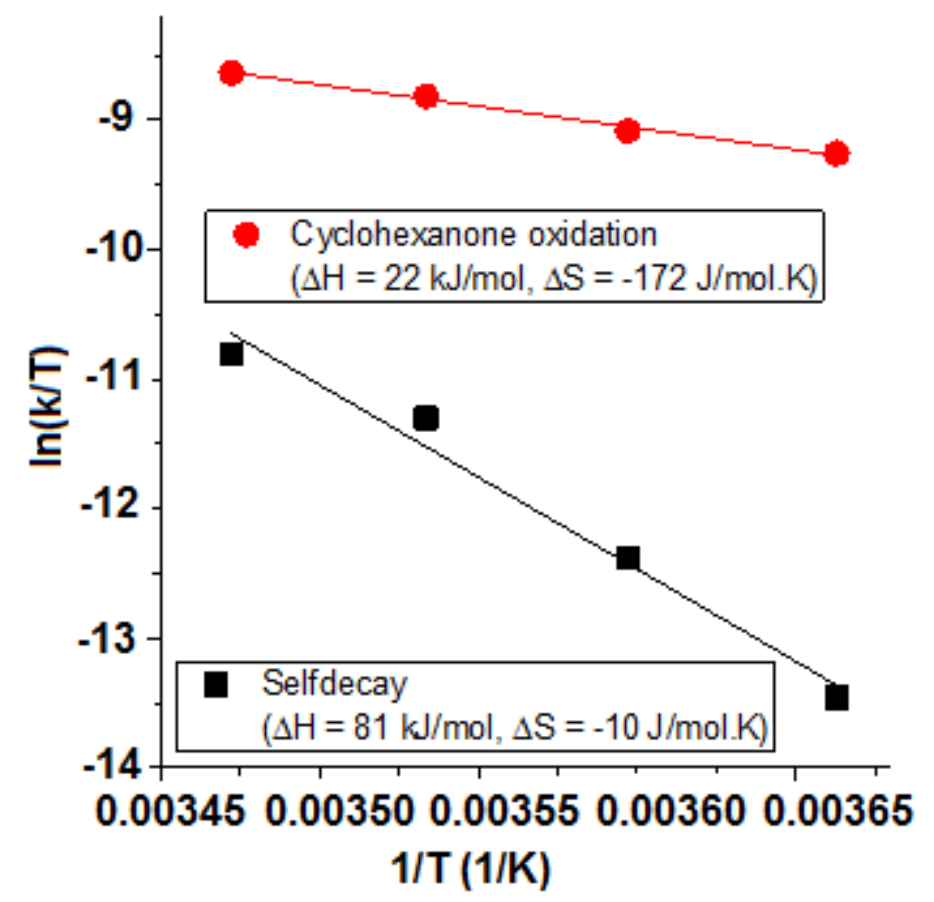

Figure S2a. Eyring plots for the self-decay of $\mathbf{2}$ (black squares) and the reaction of $\mathbf{2}$ with cyclohexanone (red circles).

\section{BVO-product (caprolactone)}

\section{Library:}

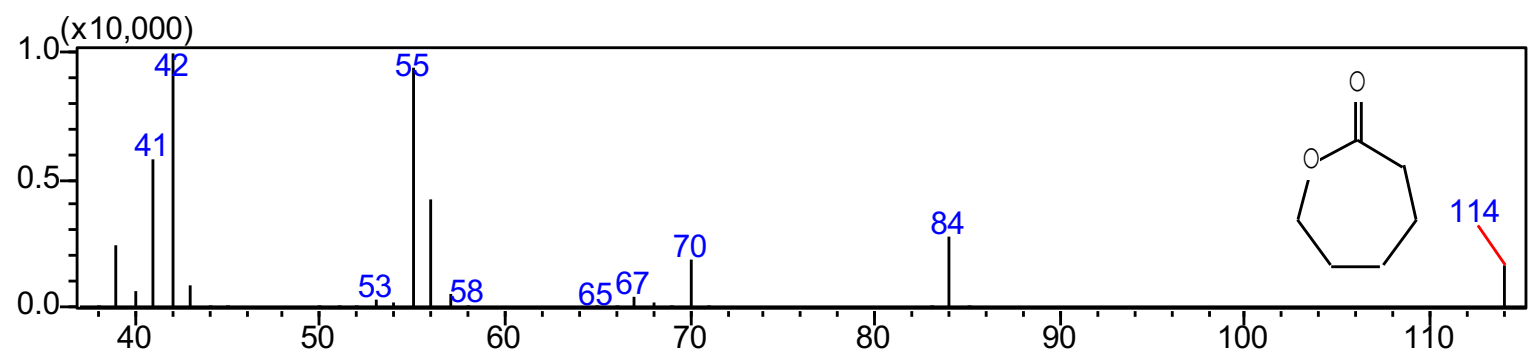

Measured:

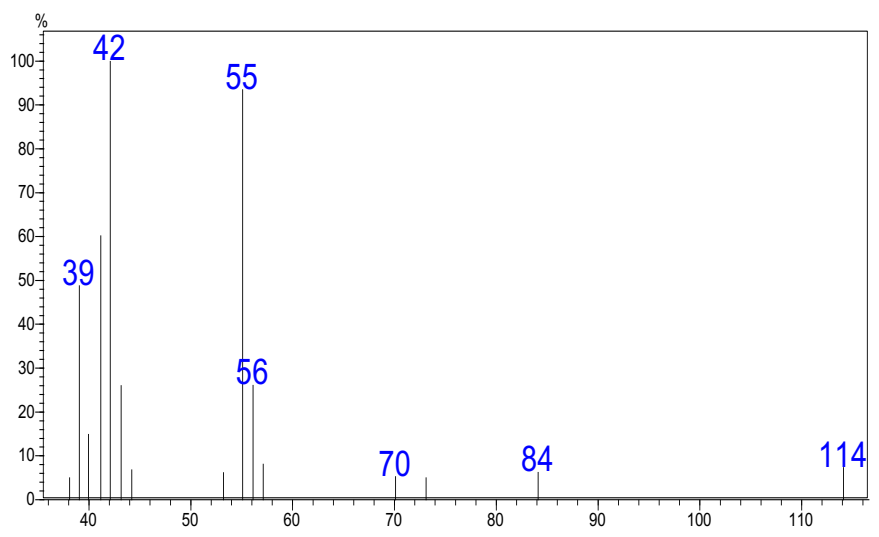




\begin{tabular}{|r|r|r|}
\hline \multicolumn{1}{|l|}{$\mathrm{m} / \mathrm{z}$} & $\begin{array}{l}\text { Absolute } \\
\text { Intensity }\end{array}$ & $\begin{array}{l}\text { Relative } \\
\text { Intensity }\end{array}$ \\
\hline 38.1 & 5540 & 6.99 \\
\hline 39.1 & 45422 & 57.35 \\
\hline 40.1 & 10900 & 13.76 \\
\hline 41.15 & 54526 & 68.84 \\
\hline 42.15 & 79207 & 100 \\
\hline 43.2 & 6818 & 8.61 \\
\hline 51.15 & 2943 & 3.72 \\
\hline 53.15 & 6974 & 8.8 \\
\hline 55.15 & 71335 & 90.06 \\
\hline 56.15 & 22697 & 28.66 \\
\hline 57.1 & 4006 & 5.06 \\
\hline 70.2 & 6953 & 8.78 \\
\hline 84.2 & 6164 & 7.78 \\
\hline 114.15 & 4183 & 5.28 \\
\hline
\end{tabular}
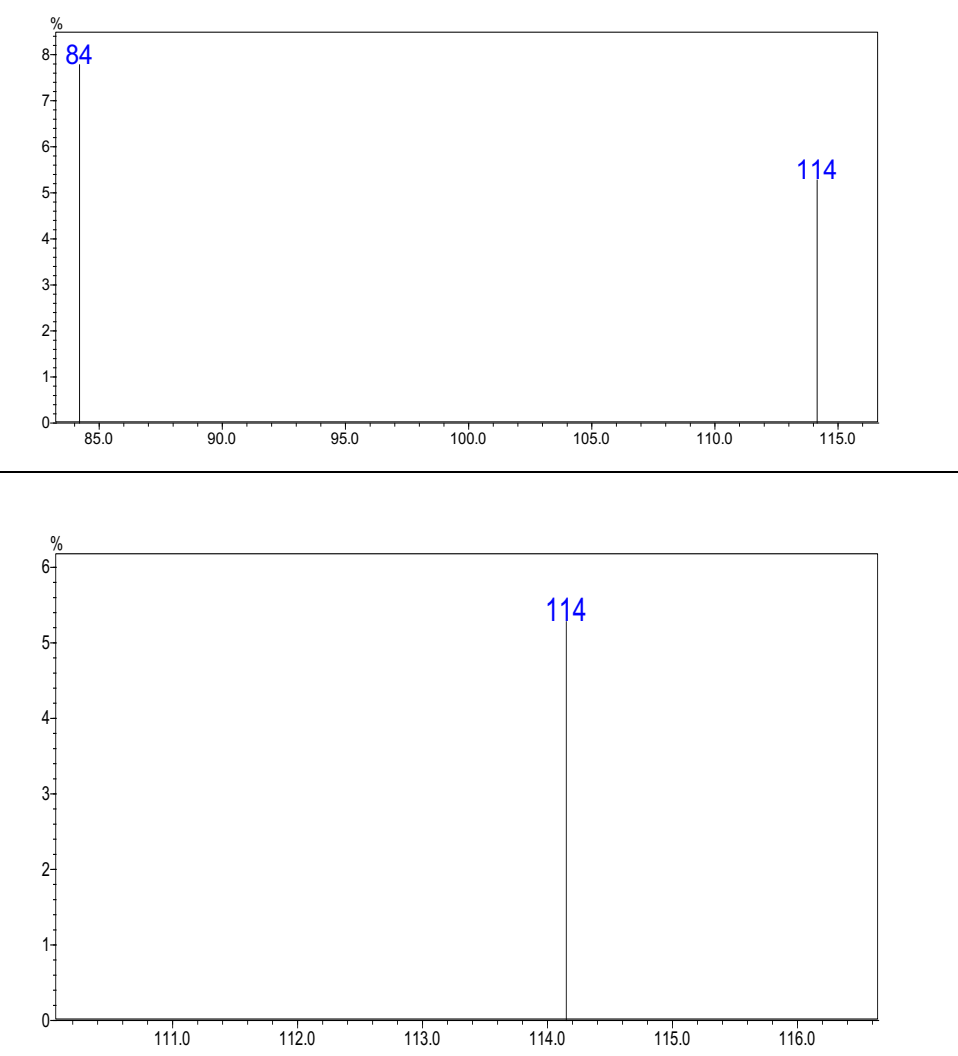

Figure S2b: Mass spectral data for the Baeyer-Villager oxidation product (caprolactone) obtained by carrying out the reaction in the presence of $\mathrm{H}_{2}{ }^{18} \mathrm{O}$, showing no ${ }^{18} \mathrm{O}$ label incorporation into the molecular ion peak at $\mathrm{m} / \mathrm{z} 114$ 


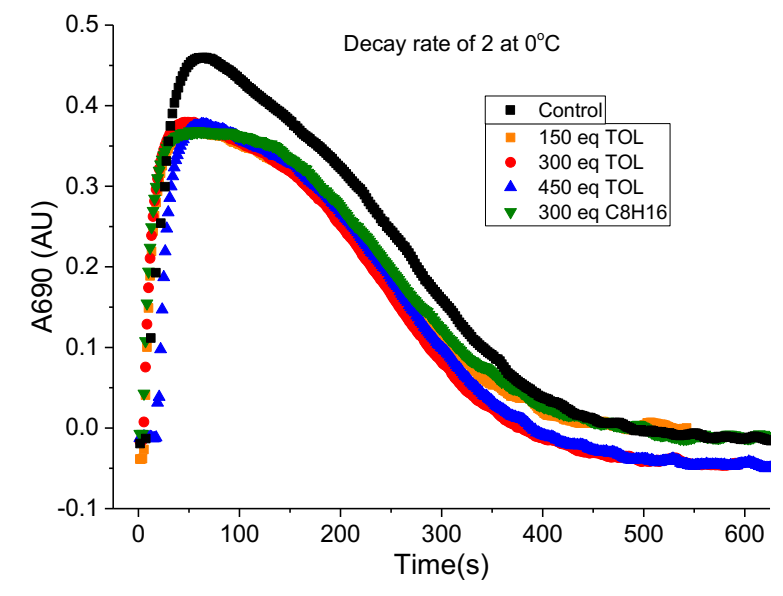

(a)

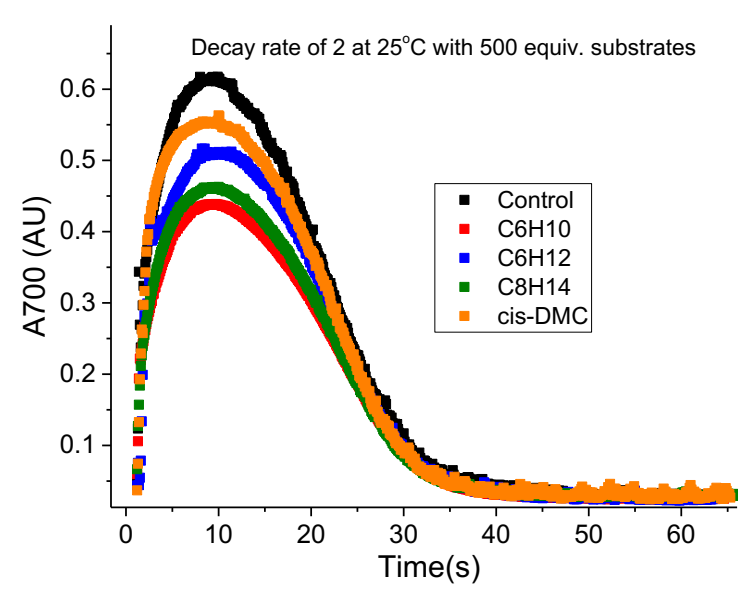

(b)

Figure S3. Time traces for the formation and decay of $\mathbf{2}$ in the presence of various substrates. Intermediate $\mathbf{2}$ was generated from the reaction of $1 \mathrm{mM} \mathbf{1}$ and either 4 equiv. $\mathrm{H}_{2} \mathrm{O}_{2}$ at $0{ }^{\circ} \mathrm{C}(\mathrm{a})$, or 25 equiv. $\mathrm{H}_{2} \mathrm{O}_{2}$ at $25{ }^{\circ} \mathrm{C}$ (b). 
Table S2: Kinetic data for hydrocarbon oxidations by 1 at $25^{\circ} \mathrm{C}$ in $\mathrm{MeCN}$.

\begin{tabular}{|c|c|c|c|c|c|c|}
\hline Substrate & $\begin{array}{l}{[2]^{\mathrm{a}}} \\
\left(10^{-3} \mathrm{M}\right)\end{array}$ & $\begin{array}{l}{\left[\mathrm{H}_{2} \mathrm{O}_{2}\right]} \\
(\mathrm{M})\end{array}$ & $\begin{array}{l}\text { [Substrate] } \\
\text { (M) }\end{array}$ & $\begin{array}{l}{ }^{b} k_{o b s} \\
\left(10^{-6} \mathrm{~s}^{-1}\right)\end{array}$ & $\begin{array}{l}{ }^{b} k_{3} \\
\left(M^{-2} s^{-1}\right)\end{array}$ & $\begin{array}{l}V^{c} \\
\left(10^{-7} \mathrm{M} \mathrm{s}^{-1}\right)\end{array}$ \\
\hline \multirow[t]{7}{*}{ DHA } & 0.05 & 0.025 & 0.1 & 13.5 & 10.8 & 13.50 \\
\hline & 0.05 & 0.05 & 0.1 & 24.6 & 9.84 & 24.60 \\
\hline & 0.05 & 0.1 & 0.1 & 47.8 & 9.56 & 47.80 \\
\hline & 0.05 & 0.025 & 0.01 & 15.9 & 12.72 & 1.59 \\
\hline & 0.05 & 0.025 & 0.025 & 16.1 & 12.88 & 4.03 \\
\hline & 0.05 & 0.025 & 0.053 & 16.1 & 12.88 & 8.53 \\
\hline & 0.05 & 0.025 & 0.075 & 15.8 & 12.64 & 11.85 \\
\hline \multirow[t]{3}{*}{ Fluorene } & 0.05 & 0.025 & 0.1 & 6.6 & 5.28 & 6.60 \\
\hline & 0.05 & 0.05 & 0.1 & 15.2 & 6.08 & 15.20 \\
\hline & 0.05 & 0.1 & 0.1 & 29.4 & 5.88 & 29.40 \\
\hline \multirow[t]{3}{*}{$\left(\mathrm{C}_{6} \mathrm{H}_{5}\right)_{3} \mathrm{CH}$} & 0.05 & 0.025 & 0.1 & 3.86 & 3.09 & 3.86 \\
\hline & 0.05 & 0.05 & 0.1 & 5.61 & 2.24 & 5.61 \\
\hline & 0.05 & 0.1 & 0.1 & 14.4 & 2.88 & 14.40 \\
\hline \multirow[t]{7}{*}{$\mathrm{C}_{6} \mathrm{H}_{5} \mathrm{CH}\left(\mathrm{CH}_{3}\right)_{2}$} & 0.05 & 0.025 & 0.1 & 2.64 & 2.11 & 2.64 \\
\hline & 0.05 & 0.05 & 0.1 & 5.58 & 2.23 & 5.58 \\
\hline & 0.05 & 0.1 & 0.1 & 10.8 & 2.16 & 10.80 \\
\hline & 0.05 & 0.025 & 0.05 & 2.54 & 2.03 & 1.27 \\
\hline & 0.05 & 0.025 & 0.075 & 2.55 & 2.04 & 1.91 \\
\hline & 0.05 & 0.025 & 0.125 & 2.55 & 2.04 & 3.19 \\
\hline & 0.05 & 0.025 & 0.15 & 2.25 & 1.80 & 3.38 \\
\hline \multirow[t]{3}{*}{$\mathrm{C}_{6} \mathrm{H}_{5} \mathrm{CH}_{2} \mathrm{CH}_{3}$} & 0.05 & 0.025 & 0.1 & 0.72 & 0.58 & 0.72 \\
\hline & 0.05 & 0.05 & 0.1 & 1.63 & 0.65 & 1.63 \\
\hline & 0.05 & 0.1 & 0.1 & 3.72 & 0.74 & 3.72 \\
\hline \multirow[t]{10}{*}{$\mathrm{C}_{6} \mathrm{H}_{5} \mathrm{CH}_{3}$} & 0.05 & 0.025 & 0.1 & 0.45 & 0.36 & 0.45 \\
\hline & 0.025 & 0.025 & 0.1 & 0.21 & 0.34 & 0.21 \\
\hline & 0.075 & 0.025 & 0.1 & 0.59 & 0.32 & 0.59 \\
\hline & 0.10 & 0.025 & 0.1 & 0.85 & 0.34 & 0.85 \\
\hline & 0.05 & 0.05 & 0.1 & 0.88 & 0.35 & 0.88 \\
\hline & 0.05 & 0.1 & 0.1 & 1.62 & 0.32 & 1.62 \\
\hline & 0.05 & 0.025 & 0.05 & 0.52 & 0.42 & 0.26 \\
\hline & 0.05 & 0.025 & 0.075 & 0.45 & 0.36 & 0.34 \\
\hline & 0.05 & 0.025 & 0.125 & 0.45 & 0.36 & 0.56 \\
\hline & 0.05 & 0.025 & 0.15 & 0.49 & 0.39 & 0.74 \\
\hline \multirow[t]{3}{*}{$\mathrm{C}_{6} \mathrm{H}_{12}$} & 0.05 & 0.025 & 0.1 & 0.23 & 0.18 & 0.23 \\
\hline & 0.05 & 0.05 & 0.1 & 0.45 & 0.18 & 0.45 \\
\hline & 0.05 & 0.1 & 0.1 & 0.9 & 0.18 & 0.90 \\
\hline \multirow[t]{3}{*}{$\mathrm{C}_{8} \mathrm{H}_{16}$} & 0.05 & 0.025 & 0.1 & 0.27 & 0.22 & 0.27 \\
\hline & 0.05 & 0.05 & 0.1 & 0.59 & 0.24 & 0.59 \\
\hline & 0.05 & 0.1 & 0.1 & 1.23 & 0.25 & 1.23 \\
\hline $\mathrm{C}_{6} \mathrm{H}_{5} \mathrm{CH}_{3}$ & 0.5 & 0.25 & 0.1 & 46.4 & 0.37 & 46.4 (KIE) \\
\hline $\mathrm{C}_{6} \mathrm{D}_{5} \mathrm{CD}_{3}$ & 0.5 & 0.25 & 0.1 & 1.24 & 0.01 & 1.24 (KIE) \\
\hline
\end{tabular}


${ }^{\text {a }}$ Generated in situ from complex 1

${ }^{\mathrm{b}}$ Normalized with the number of equivalent $\mathrm{C}-\mathrm{H}$ bonds $(\mathrm{n})$ on the substrate

${ }^{c} \mathrm{~V}=k_{o b s} \times[\mathrm{S}] ; k_{o b s}$ was extracted from a plot of $\ln \left[\text { substrate] }{ }_{t} \text { (calculated as [substrate] }{ }_{t}=\text { [substrate] }\right]_{0}$

$-\left[\right.$ product $_{t}$ ) vs. time and normalized with the number of hydrogens that could be cleaved.

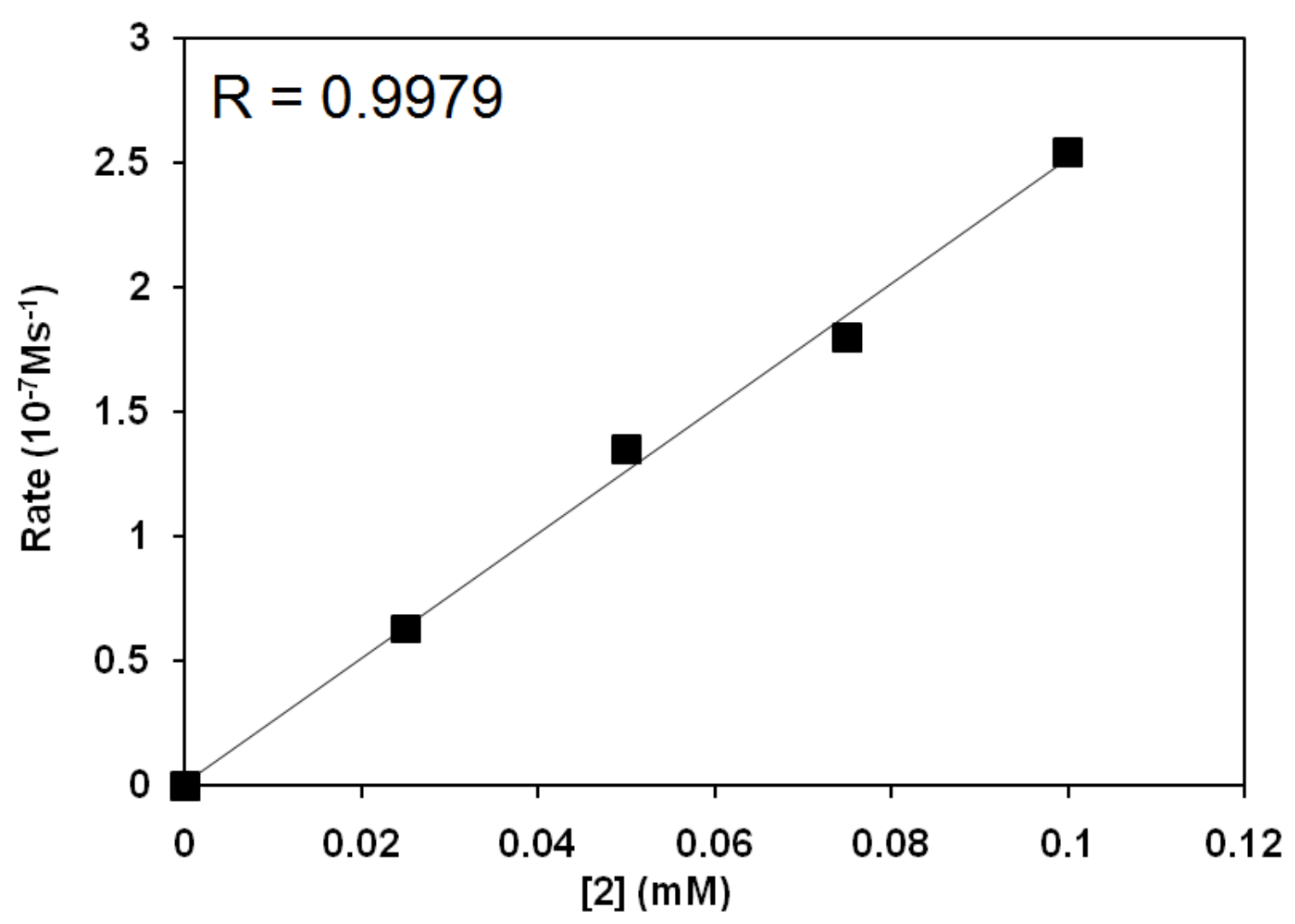

Figure S4. The rate of the catalytic oxidation of toluene plotted against the initial

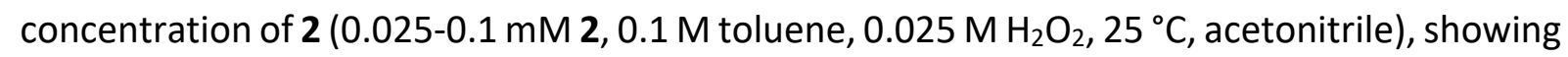
that the reaction rate increases proportionally to [catalyst]. 


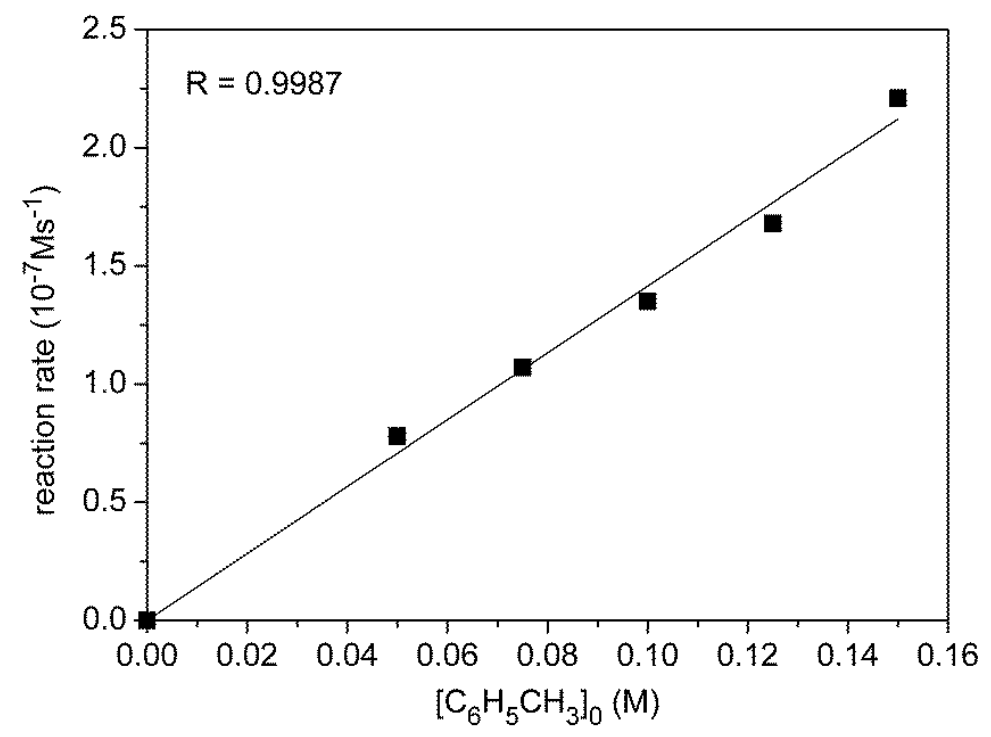

Figure S5. The rates of the catalytic oxidation of toluene plotted against the initial concentration of toluene ( $0.05 \mathrm{mM} \mathrm{2,} \mathrm{0.05-0.15} \mathrm{M} \mathrm{toluene,} 0.025 \mathrm{M} \mathrm{H}_{2} \mathrm{O}_{2}, 25{ }^{\circ} \mathrm{C}$, acetonitrile), showing that the reaction rate increases proportionally to [toluene]

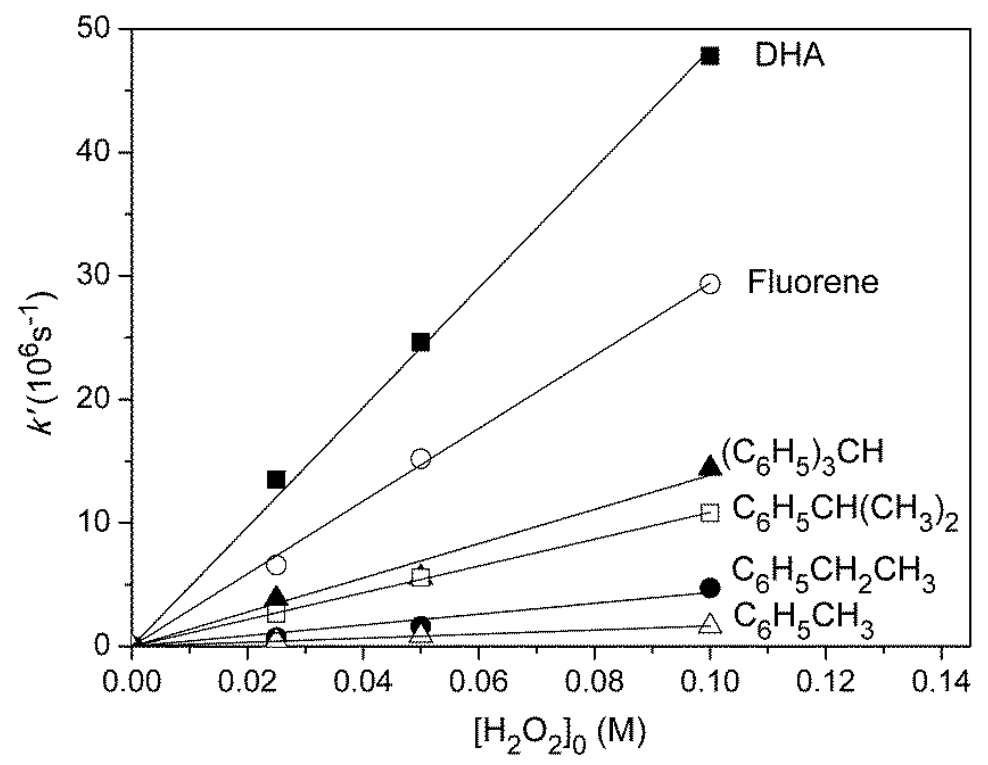

Figure S6. The dependence of $\mathrm{k}^{\prime}$ on the initial hydrogen peroxide concentrations for the

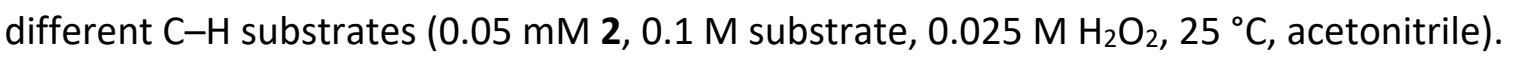




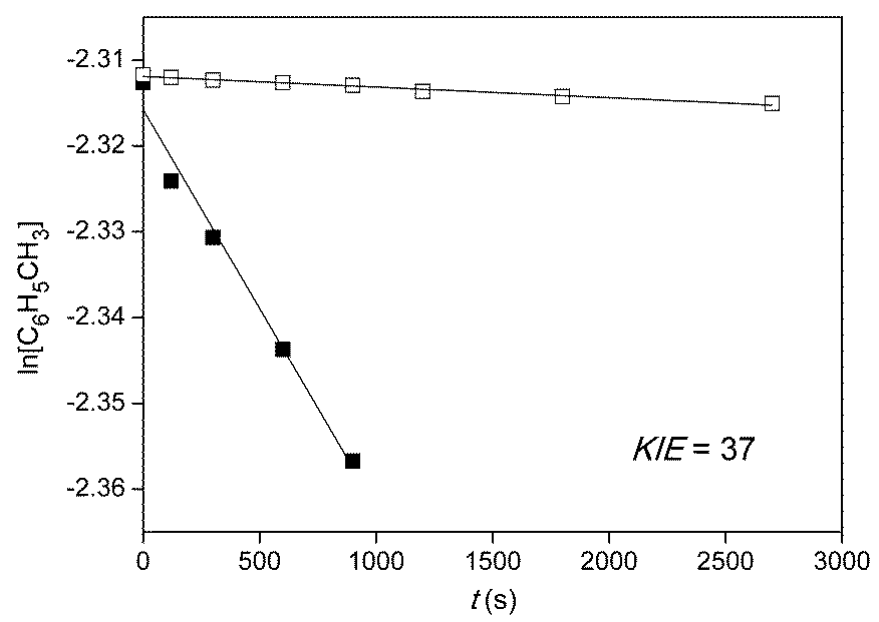

Figure S7. Difference between the reaction rates for toluene and $\mathrm{d}^{8}$-toluene oxidation under identical conditions ( $1 \mathrm{mM} \mathrm{1,} 0.1 \mathrm{M}$ toluene, $0.25 \mathrm{M} \mathrm{H}_{2} \mathrm{O}_{2}, 25^{\circ} \mathrm{C}$, acetonitrile).

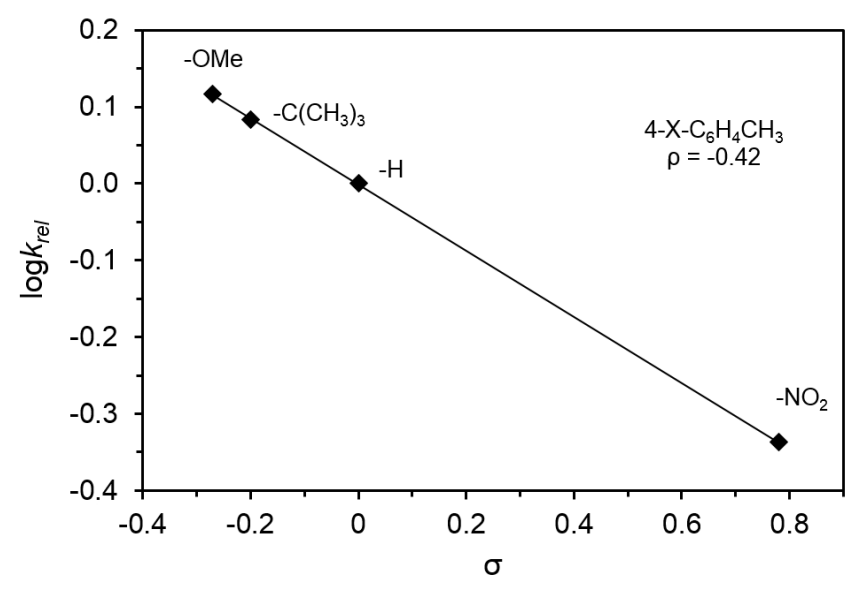

Fig. S8. Hammett plot for the catalytic oxidation of para-substituted toluenes. The $k_{\text {rel }}$ values were: $\mathrm{X}=\mathrm{H}, 1.00 ; \mathrm{X}=\mathrm{OMe}, 1.31 ; \mathrm{X}=\mathrm{C}\left(\mathrm{CH}_{3}\right)_{3}, 1.21 ; \mathrm{X}=\mathrm{NO}_{2}, 0.46$. Reaction conditions: substrates $(1.0 \mathrm{mmol}), 1(0.01 \mathrm{mmol}), \mathrm{H}_{2} \mathrm{O}_{2}(2.5 \mathrm{mmol})$ in $10 \mathrm{~mL}$ acetonitrile under $\mathrm{Ar}$ at 25 ${ }^{\circ} \mathrm{C}$. 


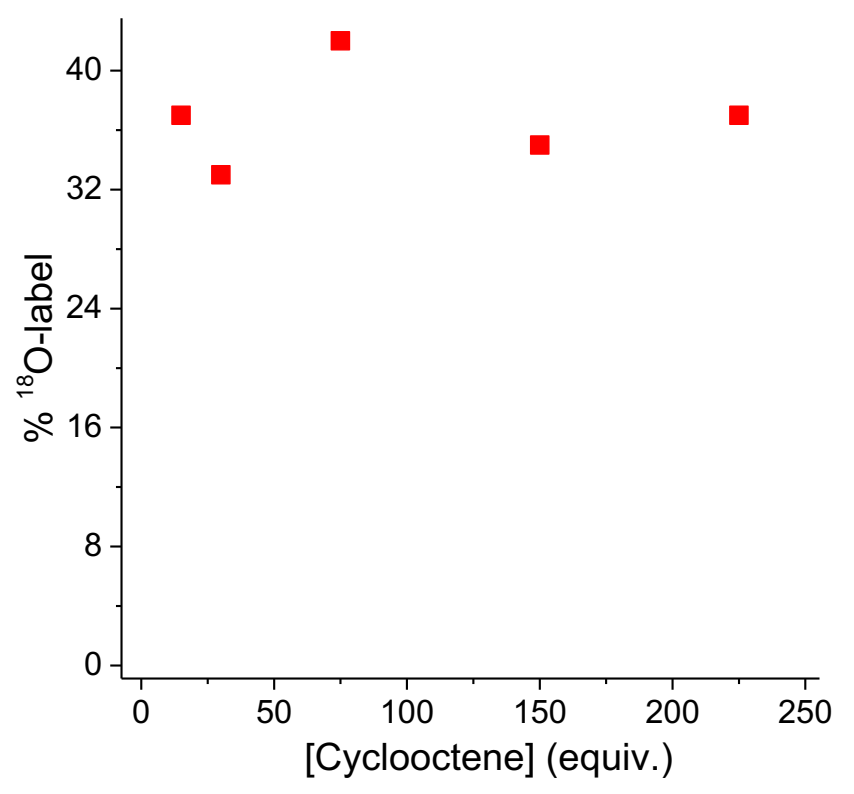

Figure S9. ${ }^{18} \mathrm{O}$ labelling of cyclooctene oxide in the oxidation of cyclooctene by $4 \mathrm{mM} 1$ and 5 equiv. $\mathrm{H}_{2} \mathrm{O}_{2}$ in the presence of $\mathrm{H}_{2}{ }^{18} \mathrm{O}$ (250 equiv.) at $25{ }^{\circ} \mathrm{C}$. The amounts of added cyclooctene (relative to $\left[\mathrm{Fe}^{\| \prime}\right]$ ) were varied. 